$008-009$

Noticias

y comentarios

PH48 - Abril 2004

\section{Red INTERLOCAL de ciudades iberoamericanas para la cultura}

En el marco de la tercera edición del Campus Euroamericano de Cooperación Cultural (Sevilla, 1 al 5 de diciembre de 2003), organizado por la Junta de Andalucía, la Organización de Estados Iberoamericanos para la Educación, la Ciencia y la Cultura (OEI) y la Fundación Interarts (Véase PH46, p. 9), se presentó la Red INTERLOCAL de ciudades iberoamericanas para la cultura.

La Red INTERLOCAL se constituyó formalmente en marzo de 2003 en la ciudad de Montevideo (Uruguay), como resultado del encuentro del I Foro INTERLOCAL de responsables de cultura de ciudades de Iberoamérica, en el que participaron 32 ciudades y administraciones territoriales provenientes de 16 paises iberoamericanos.

El objetivo de conformar una red de cooperación local-local es intercambiar experiencias y modalidades de gestión de cara a las complejidades que la cultura presenta en las ciudades contemporáneas. Asimismo, generar nuevos espacios de cooperación, tanto observatorios y laboratorios centrados en el análisis e implementación de políticas culturales de base, como organismos transversales posibilitadores de proyectos de cooperación inter e intra-ciudades. El trabajo de la red estará fundamentalmente basado en dos líneas básicas: información y formación.

Entre los acuerdos adoptados en la reunión de Montevideo cabe destacar:

$>$ El establecimiento de un encuentro bienal con el nombre de Foro INTERLOCAL de ciudades iberoamericanas para la cultura, cuya próxima edición tendrá lugar en la ciudad de Quito, Ecuador, durante el mes de marzo de 2005.
> La Comisión Gestora creada al efecto, integrada por autoridades municipales de Barcelona, Bogotá, Curitiba, La Paz, Managua, Montevideo y Quito, se reunirá en el mes de mayo de 2004 en BarceIona y el plenario de la Red tendrá lugar en Quito en marzo de 2005, coincidiendo con la celebración del II Foro INTERLOCAL.

$>$ Elaboración de una página web de la Red y de una base de datos de ciudades iberoamericanas para la cultura que tenga como función sistematizar y difundir las políticas, estructuras y programas de las ciudades miembros.

$>$ Creación de dos grupos de trabajo, uno de ellos orientado a elaboración de sistemas de información para la toma de decisiones en materia de política cultural local (indicadores, evaluación, etc.) y el otro, a la formación de formadores y administradores culturales locales en cooperación con la Red de Centros y Unidades de Formación en Gestión Cultural, IBERFORMAT, promovida por la Fundación Interarts y la OEI.

> Finalmente, la Red INTERLOCAL dará apoyo al proceso de la Agenda 21 de la Cultura promovida por el IV Foro Mundial de Autoridades Locales para la Inclusión Social, que se celebrará en Barcelona en el mes de mayo de 2004 en el marco del Forum Universal de las Culturas.

La Diputación de Barcelona asumirá la secretaría ejecutiva de la Red para este primer periodo y contará con el auspicio y apoyo de la Organización de Estados Iberoamericanos en el marco del convenio firmado entre ambas instituciones.

Más información:

Web: www.campus-oei.org/interlocal/declaracion.htm

Correo-e.: interlocal@diba.es

\title{
Un taller SIRCHAL en Ciudad Bolívar aborda la socialización del patrimonio cultural
}

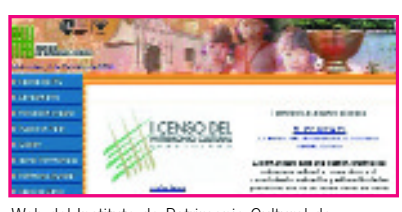

Web del Instituto de Patrimonio Cultural de Venezuela: www.ipc.gov.ve
En el marco del Convenio interinstitucional entre la Gobernación del Estado Bolívar y el Instituto del Patrimonio Cultural de Venezuela, con el auspicio de la Comisión Ministerial y del Viceministerio de Cultura para la candidatura de Ciudad Bolivar en la angostura del Orinoco como Patrimonio Mundial, se realizaron en Ciudad Bolivar, en octubre de 2003, un conjunto de actividades orientadas a profundizar en el conocimiento de problemas vinculados a la limitada identidad y valoración de las riquezas culturales y patrimoniales de la región, por parte de la comunidad involucrada y a fortalecer capacidades sociales y técnicas favorables a la Socialización del patrimonio cultural de este paisaje cultural.
En este contexto, se ha entendido por Socialización el conjunto de procesos de apropiación social que se requieren para garantizar la concienciación, la valoración y la conservación adecuada de las expresiones y significados de importancia patrimonial, existentes en la unidad del paisaje humanizado, objeto de esta propuesta. En este sentido, se ha hecho especial énfasis en cuanto a que dichas expresiones y significados patrimoniales constituyen potenciales fundamentos y recursos para la implementación de experiencias de desarrollo socioeconómico al servicio de los ciudadanos y comunidades vinculados.

Sobre este fundamento se consideró la necesidad de elaborar un Taller SIRCHAL (Seminario Interna- 
cional de Centros Históricos de América Latina) a fin de que esta experiencia permitiera una aproximación más precisa de la Socialización del patrimonio cultural de este paisaje cultural. Con anterioridad al taller se abordó el tema de la socialización -tanto como proceso educativo, comunitario, así como recurso de desarrollo socioeconómico- en unas jornadas diseñadas para la identificación de problemas, objetivos y alternativas, discusión de ideas, búsqueda de consensos y sistematización de acuerdos.

En la ejecución del taller SIRCHAL en Ciudad Bolívar se contó con representantes internacionales de reconocidas instituciones afines a la gestión del patrimonio cultural, asi como con autoridades nacionales y regionales. Sobre la plataforma conceptual de que la socialización del patrimonio cultural sólo es posible si se aborda de manera complementaria, el taller debatió las tres aristas que conforman dicha socialización: conocer, conservar y valorar. Continuas reflexiones sobre estos tres aspectos tuvieron como balance general la idea de que el conocimiento, defensa, conservación y aprecio de los valores patrimoniales, a la vez que el desarrollo de sus potencialidades en tanto recursos para alcanzar una mejor calidad de vida, debe ser el fin último en la gestión patrimonial del paisaje cultural de Ciudad Bolivar en la angostura del Orinoco. Estas ideas se expresan en un documento final, suscrito por las autoridades locales, regionales y nacionales, y en el que, con el apoyo de la ciudadanía, se estableció un frente común para su consecución.

\section{José Manuel Rodriguez}

Presidente del Instituto del Patrimonio

Cultural de Venezuela

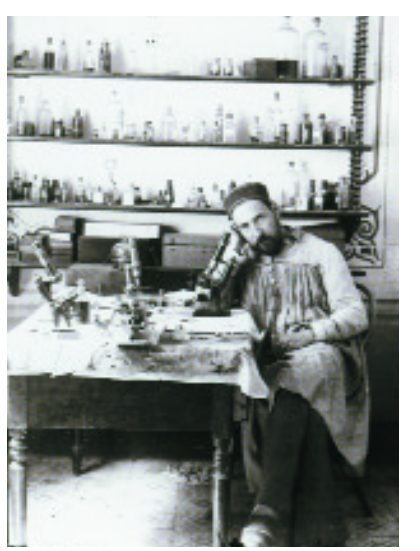

Autorretrato de Cajal en su laboratorio de Valencia, h.1885. Copia digital de placa al gelatino-bromuro, $9^{\prime} 9 \times 6^{\prime} 3 \mathrm{~cm}$. Imagen procedente del catálogo Santiago Ramón y Cajal (1852-2003). Ciencia y Arte. Madrid: La Casa Encendida, 2003, p. 75 (CAT 005)

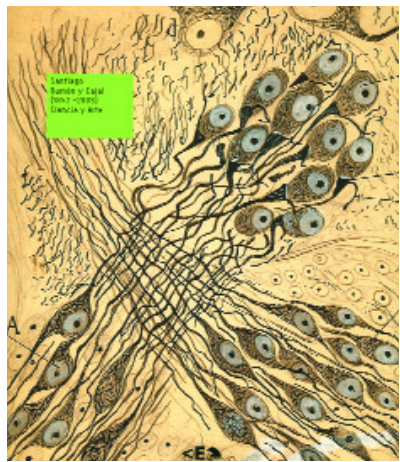

Portada del catálogo de la muestra. Santiago Ramón y Cajal (1852-2003). Ciencia y Arte. Madrid: La Casa Encendida, 2003

\section{Cajal y sus dibujos: ¿arte o ciencia?}

La aparición de Santiago Ramón y Cajal en el mundo de la neurociencia provocó un cambio radical en el curso de su historia. A diferencia de otros grandes investigadores, Cajal no hizo un solo gran descubrimiento, sino que realizó numerosas e importantes contribuciones al conocimiento de la estructura y función del sistema nervioso. Los estudios de Cajal sobre la microanatomía de virtualmente todo el sistema nervioso, sus observaciones sobre la degeneración y regeneración, junto con sus teorias sobre la función, desarrollo y plasticidad del sistema nervioso, tuvieron un profundo impacto sobre los científicos de su era. Numerosos investigadores siguieron el ejemplo establecido por Cajal, comprobando y ampliando sus teorías en prácticamente todos los campos de la neurociencia. Estos estudios representan las raices de los descubrimientos actuales en algunas de las áreas más apasionantes sobre la estructura y función del cerebro en condiciones normales y patológicas.

En los tiempos de Cajal, la microfotografía no se había desarrollado y prácticamente la única forma de ilustrar las observaciones microscópicas era mediante dibujos. Esta limitación técnica dio lugar a cierto escepticismo. Algunos dibujos de Cajal eran considerados por algunos investigadores como interpretaciones "artísticas" y no como copias más o menos exactas de las preparaciones histológicas. Sin embargo, el análisis de las preparaciones histológicas de Cajal, realizado por científicos y que hemos tenido la fortuna de examinar, ha mostrado la veracidad de estos dibujos. Por estos motivos, los dibujos de Cajal son de extraordinaria importancia no por su belleza y su indudable valor museistico, sino porque nos muestran la microorganización del sistema nervioso, como un mapa que contiene las conexiones de las neuronas y las rutas que siguen los impulsos nerviosos a través de las mismas. De esta forma, el valor científico de los dibujos de Cajal supera con creces el valor artístico.
Antes de los estudios de Cajal se conocía muy poco sobre la estructura y función del sistema nervioso, debido principalmente al hecho de que con las técnicas histológicas disponibles no era posible visualizar las neuronas en su totalidad (cuerpo celular, dendritas y axón). Sin embargo, en 1873 una verdadera revolución comenzó con el descubrimiento de la "reazione nera" (reacción negra) de Camillo Golgi (1843-1926). Por primera vez se pudieron observar, en preparaciones histológicas, a las células nerviosas con todas sus partes usando el método de Golgi. Además, sólo una pequeña proporción de las células nerviosas se teñian en una preparación histológica dada, permitiendo examinarlas individualmente. Por otra parte, esta tinción era de gran calidad, lo que permitió la caracterización de las mismas (es decir, la identificación de diversos tipos de células nerviosas), el análisis morfológico detallado y el estudio de sus posibles

Exposición "Santiago Ramón y Cajal (1852-2003). Ciencia y Arte"

Los dibujos histológicos del premio Nobel Santiago Ramón y Cajal estuvieron expuestos hasta el 5 de enero en La Casa Encendida de Madrid. A los 169 dibujos científicos, se unieron más de 90 fotografias y una serie de instrumentos científicos utilizados por Cajal.

El discurso de la muestra -primera en torno a esta producción artística- explicaba la intersección de la ciencia, la cultura y el arte. Cien años después de realizados, los dibujos realizados por Cajal se ofrecen como elementos imprescindibles para entender los cambios en la visión del arte que se produjeron a principios del siglo XX; también sus estudios sobre la técnica fotográfica para ser aplicada a la investigación científica son aportaciones irreemplazables.

Más información:

La Casa Encendida

Tel.: 915063875

Web: www.lacasaencendida.com

Correo-e.: casaencendida@cajamadrid.es 